\title{
Exponential Synchronization of Inertial Cohen-Grossberg Neural Networks with Time-Varying Delays via Periodically Intermittent Control
}

\author{
Qing Ding ${ }^{1 \#}$, Yinfang Song ${ }^{2}$ \\ ${ }^{1,2}$ School of Information and Mathematics, Yangtze University, Jingzhou 434023. Hubei, China. \\ \# corresponding author \\ Type of Review: Peer Reviewed. \\ DOl: http://dx.doi.org/10.21013/jas.v15.n2.p1

\section{How to cite this paper:} \\ Ding, Q., Song, Y. (2020). Exponential Synchronization of Inertial Cohen-Grossberg Neural \\ Networks with Time-Varying Delays via Periodically Intermittent Control. IRA International Journal \\ of Applied Sciences (ISSN 2455-4499), 15(2), 15-29. doi:http://dx.doi.org/10.21013/jas.v15.n2.p1
}

(C) Institute of Research Advances.

\section{(cc)) BY-NO}

This work is licensed under a Creative Commons Attribution-Non Commercial 4.0 International License subject to a proper citation to the publication source of the work.

Disclaimer: The scholarly papers as reviewed and published by the Institute of Research Advances (IRA) are the views and opinions of their respective authors and are not the views or opinions of the IRA. The IRA disclaims of any harm or loss caused due to the published content to any party.

Institute of Research Advances is an institutional publisher member of Publishers International Linking Association Inc. (PILA-CrossRef), USA. The institute is an institutional signatory to the Budapest Open Access Initiative. Hungary advocating the open-access of scientific and scholarly knowledge. The Institute is a registered content provider under Open Access Initiative Protocol for Metadata Harvesting (OAl-PMH).

The journal is indexed \& included in CAS Source Index of Chemical Abstracts Service of American Chemical Society (USA), Index Copernicus (IC Value 85.27), WorldCat Discovery Service (USA), CrossRef Metadata Search (USA), WorldCat (USA), OCLC (USA), Open J-Gate (India), EZB (Germany) Scilit (Switzerland), Airiti (China), Bielefeld Academic Search Engine (BASE) of Bielefeld University, Germany, PKP Index of Simon Fraser University, Canada. 


\begin{abstract}
This paper deals with the exponential synchronization problem of inertial Cohen-Grossberg neural networks with time-varying delays under periodically intermittent control. In light of Lyapunov-Krasovskii functional method and inequality techniques, some sufficient conditions are attained to ensure the exponential synchronization of the master-slave system on the basis of p-norm. Meanwhile, the periodically intermittent control schemes are designed. Finally, in order to verify the effectiveness of theoretical results, some numerical simulations are provided.
\end{abstract}

Keywords : Synchronization; Inertial Cohen-Grossberg neural networks; periodically intermittent control; delays; Lyapunov-Krasovskii functional.

\title{
1. Introduction
}

The Cohen-Grossberg Neural Network (CGNN) was first proposed by Cohen and Grossberg in 1983 [1]. Subsequently, it was widely used in various fields such as associative memory, signal processing and optimization problems. In the actual operation of the hardware of the neural network system, due to the limited signal exchange and transmission speed, time delay is inevitable, which usually destroys the stability and synchronization of the considered systems, and even causes oscillation and bifurcation. Therefore, the investigation of delayed Cohen-Grossberg neural networks (CGNNs) has aroused researcher's interests, and many enlightening results have been appeared in [2-6]. It is worth noting that a more complex system yields since inertial terms are introduced into the CGNN system, which is called as the inertial Cohen-Grossberg neural network (ICGNN). Actually, the existence of inertial terms has strong biological background [7-8] and electronic environment [9-10]. In recent years, many results about inertial Cohen-Grossberg neural networks (ICGNNs) [11-15] have been reported.

There is no doubt that synchronous behavior is widespread in the real life, and it plays a vital role in chemical reactions, medical equipment, communication and transmission. As a matter of fact, how to overcome harmful synchronization and achieve beneficial synchronization is also a significant problem of network science research. To solve this problem, a variety of effective control strategies have been adopted, including impulsive control [16], intermittent control [17], adaptive control [18] and sampling control [19] and so on. Compared with continuous control, intermittent control is more economical and efficient; plenty of research results have been developed in [20-26]. Particularly, by employing Lyapunov functional theory, mathematical induction and inequality techniques, based on p-norm, Xing et al. [20] proposed the conditions for exponential synchronization of delayed recurrent neural network by designing suitable periodic intermittent controller. Zhang and Shen [21] adopted non-smooth analysis and control theory to consider the problem of the exponential synchronization of memristor-based chaotic neural networks via periodically intermittent control. Besides, the introduction of inertial terms will cause complex dynamic behaviors. Hence, it is also of great significance to study the inertial neural networks. Under the two types of activation functions, Tang and Jian [22] obtained several delay-dependent criteria on the exponential synchronization of the inertial neural network with mixed delays by virtue of algebraic inequality and linear matrix inequality. Wan et al. [23] employed the Lyapunov-Krasovskii functional approach and Wirtinger's inequality to realize global exponential synchronization on isolated nodes for inertial reaction-diffusion coupled neural network with proportional delay via periodically intermittent control. On the other hand, some researchers used periodic intermittent control to study the synchronization of CGNNs. Based on the theory of infinite 
norms, Yu et al. [24] discussed the synchronization problem of a class of CGNNs with time delay and general amplification function under periodic intermittent control. By constructing suitable Lyapunov functional and utilizing comparison principle, Mei et al. [25] investigated the p-synchronization for stochastic CGNNs with variable coefficients and reaction-diffusion terms with periodic intermittent control. Hui et al. [26] designed a new hybrid control strategy which consists of pinning control and periodic intermittent control, and obtained some new synchronization conditions for memristive CGNNs with mixed delays. However, up to now, the problem of exponential synchronization of the ICGNNs with periodic intermittent control has not been considered.

Inspired by the above discussions, in this paper, we focus on exponential synchronization of ICGNNs with time-varying delays via periodically intermittent control. The main contributions of this article are the following three aspects: (1) Since inertial terms are introduced to the CGNNs, our considered system become more complex. (2) In order to realize the synchronization of ICGNNs, periodically intermittent controller is designed and feedback gain is obtained. (3) Combing Lyapunov-Krasovskii functional method and inequality techniques, some sufficient conditions are given to guarantee the exponential stability of error system based on the p-norm.

This paper is organized as follows. In Section 2, some preliminaries and model descriptions are given. Then, some sufficient conditions are derived to ensure the exponential synchronization in Section 3.Finally, a numerical example is provided in Section 4 to verify the feasibility of the results.

\section{Preliminaries}

Consider a class of inertial Cohen-Grossberg with time-varying delay as follows

$$
\begin{array}{r}
\frac{d^{2} x_{i}(t)}{d t^{2}}=-u_{i} \frac{d x_{i}(t)}{d t}-v_{i}\left(x_{i}(t)\right)\left[b_{i}\left(x_{i}(t)\right)-\sum_{j=1}^{n} c_{i j} f_{j}\left(x_{j}(t)\right)\right. \\
\left.-\sum_{j=1}^{n} d_{i j} f_{j}\left(x_{j}(t-\sigma(t))\right)-I_{i}\right], i, j \in \Lambda,
\end{array}
$$

where $\Lambda=\{1,2, \cdots, n\}, u_{i}>0$ are constants, the second-order derivative is referred to as an inertial term of $(1), x_{i}(t)$ corresponds to the state variable of the ith neuron at the time $t, v_{i}(\cdot)$ represents an amplification function; $b_{i}(\cdot)$ is an appropriate behaved function, $c_{i j}$ and $d_{i j}$ are constants which denote the connection strengths of the neural networks; $f_{j}(\cdot)$ denotes the activation function of $j t h$ neuron; $\sigma(t)$ corresponds to the transmission delay; $I_{i}$ is the external input.

The initial conditions of system (1) are

$$
x_{i}(s)=\varphi_{i}(s), \frac{d x_{i}(s)}{d t}=\varphi_{i}^{\prime}(s), s \in[-\sigma, 0], i \in \Lambda,
$$

Where $\varphi_{i}(s)$ and $\varphi_{i}^{\prime}(s)$ are bounded and continuous functions.

Set variable transformation $\frac{d x_{i}(t)}{d t}=-x_{i}(t)+y_{i}(t), i \in \Lambda$, the systems (1) can be rewritten as

$$
\left\{\begin{aligned}
\frac{d x_{i}(t)}{d t} & =-x_{i}(t)+y_{i}(t) \\
\frac{d y_{i}(t)}{d t} & =-\left(1-u_{i}\right) x_{i}(t)-\left(u_{i}-1\right) y_{i}(t)-v_{i}\left(x_{i}(t)\right)\left[b_{i}\left(x_{i}(t)\right)\right. \\
& \left.-\sum_{j=1}^{n} c_{i j} f_{j}\left(x_{j}(t)\right)-\sum_{j=1}^{n} d_{i j} f_{j}\left(x_{j}(t-\sigma(t))\right)-I_{i}\right]
\end{aligned}\right.
$$


with initial condition

$$
x_{i}(s)=\varphi_{i}(s), y_{i}(s)=x_{i}(s)+\frac{d x_{i}(s)}{d t}=\varphi_{i}(s)+\varphi_{i}^{\prime}(s)=\psi_{i}(t), s \in[-\sigma, 0], i \in \Lambda .
$$

Let system (2) be master system, and the corresponding slave system can be expressed as

$$
\left\{\begin{aligned}
\frac{d z_{i}(t)}{d t}= & -z_{i}(t)+w_{i}(t)+K_{1 i} \\
\frac{d w_{i}(t)}{d t}= & -\left(1-u_{i}\right) z_{i}(t)-\left(u_{i}-1\right) w_{i}(t)-v_{i}\left(z_{i}(t)\right)\left[b_{i}\left(z_{i}(t)\right)\right. \\
& \left.-\sum_{j=1}^{n} c_{i j} f_{j}\left(z_{j}(t)\right)-\sum_{j=1}^{n} d_{i j} f_{j}\left(z_{j}(t-\sigma(t))\right)-I_{i}\right]+K_{2 i}
\end{aligned}\right.
$$

where $z_{i}(t), w_{i}(t)$ are state variables of the slave system, and $K_{1 i}, K_{2 i}$ are the periodically intermittent controllers represented by

$$
\left\{\begin{array}{rr}
K_{1 i}=k_{1 i}\left(z_{i}(t)-x_{i}(t)\right), K_{2 i}=k_{2 i}\left(w_{i}(t)-y_{i}(t)\right) \quad m T \leq t<(m+\theta) T, \\
K_{1 i}=K_{2 i}=0, & (m+\theta) T \leq t<(m+1) T,
\end{array}\right.
$$

where $m=0,1,2 \cdots, i \in \Lambda, k_{1 i}, k_{2 i}$ denote the control strength, $T(T>0)$ representscontrol period, $\theta(0<\theta<1)$ is known as the width index of the control. And the initial conditions are shown as

$$
\begin{aligned}
z_{i}(s)= & \tilde{\varphi}_{i}(s), \\
& w_{i}(s)=\frac{d z_{i}(s)}{d t}+z_{i}(s)=\tilde{\varphi}_{i}^{\prime}(s)+\tilde{\varphi}_{i}(s)=\tilde{\psi}_{i}(s), s \in[-\sigma, 0], i \in \Lambda,
\end{aligned}
$$

where $\tilde{\varphi}_{i}(s)$ and $\tilde{\psi}_{i}(s)$ are bounded and continuous functions.

Let $e_{1 i}(t)=z_{i}(t)-x_{i}(t), e_{2 i}(t)=w_{i}(t)-y_{i}(t), i \in \Lambda$, we can get the error system as follows

$$
\left\{\begin{aligned}
\frac{d e_{1 i}(t)}{d t} & =-e_{1 i}(t)+e_{2 i}(t)+K_{1 i} \\
\frac{d e_{2 i}(t)}{d t} & =-\left(1-u_{i}\right) e_{1 i}(t)-\left(u_{i}-1\right) e_{2 i}(t) \\
& -\left[v_{i}\left(z_{i}(t)\right) b_{i}\left(z_{i}(t)\right)-v_{i}\left(x_{i}(t)\right) b_{i}\left(x_{i}(t)\right)\right] \\
& +v_{i}\left(z_{i}(t)\right)\left[\sum_{j=1}^{n} c_{i j} \bar{f}_{j}\left(e_{1 j}(t)\right)+\sum_{j=1}^{n} d_{i j} \bar{f}_{j}\left(e_{1 j}(t-\sigma(t))\right)\right] \\
& +v_{i}\left(e_{1 i}(t)\right)\left[\sum_{j=1}^{n} c_{i j} f_{j}\left(x_{j}(t)\right)+\sum_{j=1}^{n} d_{i j} f_{j}\left(x_{j}(t-\sigma(t))\right)+I_{i}\right]+K_{2 i}
\end{aligned}\right.
$$

where

$$
\begin{aligned}
& \bar{f}_{j}\left(e_{1 j}(t)\right)=f_{j}\left(z_{j}(t)\right)-f_{j}\left(x_{j}(t)\right), \\
& \quad \bar{f}_{j}\left(e_{1 j}(t-\sigma(t))\right)=f_{j}\left(z_{j}(t-\sigma(t))\right)-f_{j}\left(x_{j}(t-\sigma(t))\right) .
\end{aligned}
$$

In order to study the exponential synchronization of system (2) and system (3) with periodically controller (4), we give the following hypotheses, lemmas and definitions.

$\left(\mathrm{H}_{1}\right)$ Suppose that time-varying delay $\sigma(t)$ are continuous and derivable, and there exist constants 
$\sigma>0, \sigma_{1}>0$ such that $0 \leq \sigma(t) \leq \sigma, \dot{\sigma}(t) \leq \sigma_{1}<1$

$\left(\mathrm{H}_{2}\right)$ For any $x, z \in R$, suppose that $0 \leq v_{i}(x) \leq \bar{v}_{i}$, and there exists constants $s_{i}>0$ satisfying

$$
\left|v_{i}(x)-v_{i}(z)\right| \leq s_{i}|x-z|, i \in \Lambda .
$$

$\left(\mathrm{H}_{3}\right)$ Suppose that the activation functions are bounded and satisfy the global Lipschitz condition, i.e., for any $x, z \in R$, there exists constants $m_{j}, l_{j}>0$ such that

$$
\left|f_{j}(x)\right| \leq m_{j},\left|f_{j}(x)-f_{j}(z)\right| \leq l_{j}|x-z|, j \in \Lambda .
$$

$\left(\mathrm{H}_{4}\right)$ For any $x, z \in R, x \neq z$, there are constants $h_{i}>0$ satisfying

$$
\frac{v_{i}(z) b_{i}(z)-v_{i}(x) b_{i}(x)}{z-x} \geq h_{i}, i \in \Lambda .
$$

$\left(\mathrm{H}_{5}\right)$ For every $i, j \in \Lambda, r_{i}+p k_{1 i}-\sum_{j=1}^{n} a_{j i}>0, \mu_{i}+p k_{2 i}-\sum_{j=1}^{n} \xi_{j i}>0$, where $r_{i}=1-\left|1-u_{i}\right|-h_{i}-s_{i}\left(\sum_{j=1}^{n}\left(\left|c_{i j}\right|+\left|d_{i j}\right|\right) m_{j}+\left|I_{i}\right|\right)-\sum_{j=1}^{n} \bar{v}_{j}\left|c_{j i}\right| l_{i}$,

$$
\begin{array}{r}
\mu_{i}=p\left(u_{i}-1\right)-1-(p-1) \sum_{j=1}^{n} \bar{v}_{i}\left(\left|c_{i j}\right|+\left|d_{i j}\right|\right) l_{j} \\
-(p-1)\left(\left|1-u_{i}\right|+h_{i}+s_{i}\left(\sum_{j=1}^{n}\left(\left|c_{i j}\right|+\left|d_{i j}\right|\right) m_{j}+\left|I_{i}\right|\right)\right),
\end{array}
$$

$a_{j i}=\frac{\bar{v}_{j}\left|d_{j i}\right| l_{i}}{1-\sigma_{1}}, \xi_{j i} \geq 0, \xi_{j i}$ are some appropriately chosen numbers.

Lemma 1. Let Assumption $\left(\mathrm{H}_{5}\right)$ hold. If $r_{i}+p k_{1 i}-\sum_{j=1}^{n} a_{j i}>0, \mu_{i}+p k_{2 i}-\sum_{j=1}^{n} \xi_{j i}>0$, $i, j \in \Lambda$, then there exists a positive constant $\varepsilon$ such that

$$
r_{i}+p k_{1 i}-e^{\varepsilon \sigma} \sum_{j=1}^{n} a_{j i}-\varepsilon \geq 0, \mu_{i}+p k_{2 i}-e^{\varepsilon \sigma} \sum_{j=1}^{n} \xi_{j i}-\varepsilon \geq 0, i, j \in \Lambda .
$$

Proof. We consider the following function

$$
\Pi_{i}\left(\varepsilon_{i}\right)=r_{i}+p k_{1 i}-e^{\varepsilon_{i} \sigma} \sum_{j=1}^{n} a_{j i}-\varepsilon_{i},
$$

where $\varepsilon_{i} \geq 0$ for every $i \in \Lambda$. By simple calculating, we can easily get

$$
\Pi_{i}^{\prime}\left(\varepsilon_{i}\right)=-1-\sigma e^{\varepsilon_{i} \sigma} \sum_{j=1}^{n} a_{j i} \text { and } \Pi_{i}(0)=r_{i}+p k_{1 i}-\sum_{j=1}^{n} a_{j i}>0 .
$$

In addition, $\Pi_{i}\left(\varepsilon_{i}\right)$ is continuous on $[0,+\infty)$ and $\Pi_{i}\left(\varepsilon_{i}\right) \rightarrow \infty$ as $\varepsilon_{i} \rightarrow \infty$. Hence, there exists a unique positive root $\varepsilon_{i}^{*}$ such that $\Pi_{i}\left(\varepsilon_{i}^{*}\right)=0$ and $\Pi_{i}\left(\varepsilon_{i}\right)>0$ for any $\varepsilon_{i} \in\left(0, \varepsilon_{i}^{*}\right)$. Denoting $\dot{\varepsilon}=\min _{i \in \Lambda}\left\{\varepsilon_{i}^{*}\right\}$, then

$$
\Pi_{i}(\dot{\varepsilon})=r_{i}+p k_{1 i}-e^{\dot{\varepsilon} \sigma} \sum_{j=1}^{n} a_{j i}-\dot{\varepsilon} \geq 0, i \in \Lambda .
$$

Similarly, we can obtain

$$
\Omega_{i}(\grave{\varepsilon})=\mu_{i}+p k_{2 i}-e^{\grave{\varepsilon} \sigma} \sum_{j=1}^{n} \xi_{j i}-\grave{\varepsilon} \geq 0, i \in \Lambda .
$$

Let $\varepsilon=\min \{\dot{\varepsilon}, \dot{\varepsilon}\}$, we can easily get

$$
\Pi_{i}(\varepsilon) \geq 0, \Omega_{i}(\dot{\varepsilon}) \geq 0, \quad i \in \Lambda .
$$

Lemma 2 [22]. Assume that $a, b$ are positive numbers and $p \geq 1$ is a positive integer, then the following inequality holds:

$$
p a b^{p-1} \leq a^{p}+(p-1) b^{p} .
$$

Definition 1. Master system (2) and slave system (3) with controller (4) can be exponentially synchronized, if there exist constants $\omega>0$ and $\gamma>0$ such that

$$
\|e(t)\|_{p} \leq \omega\|\Psi\|_{p} e^{-\gamma t}, t>0,
$$

where

$\|e(t)\|_{p}=\left(\sum_{i=1}^{n}\left|e_{1 i}(t)\right|^{p}+\sum_{i=1}^{n}\left|e_{2 i}(t)\right|^{p}\right)^{\frac{1}{p}}$, 
$\|\Psi\|_{p}=\left(\sum_{i=1}^{n} \sup _{-\sigma \leq s \leq 0}\left|\varphi_{i}(s)-\tilde{\varphi}_{i}(s)\right|^{p}+\sum_{i=1}^{n} \sup _{-\sigma \leq s \leq 0}\left|\psi_{i}(s)-\tilde{\psi}_{i}(s)\right|^{p}\right)^{\frac{1}{p}}$,

$p \geq 1, i \in \Lambda$.

\section{Main results}

In this section, acceptable period $T$ and $k_{1 i}, k_{2 i}$ are designed to achieve the exponential synchronization of master system (2) and slave system (3).

Theorem 1. Assume that hypotheses $\left(\mathrm{H}_{1}\right)-\left(\mathrm{H}_{5}\right)$ hold. If there exists $\varepsilon>0$ such that the following conditions are satisfied:

$$
\frac{\varepsilon}{p}-\varpi(1-\theta)>0
$$

and

$$
r_{i}+p k_{1 i}-e^{\varepsilon \sigma} \sum_{j=1}^{n} a_{j i}-\varepsilon \geq 0, \mu_{i}+p k_{2 i}-e^{\varepsilon \sigma} \sum_{j=1}^{n} \xi_{j i}-\varepsilon \geq 0, i, j \in \Lambda,
$$

where $\varpi=\max \left\{\max _{i \in \Lambda}\left\{k_{1 i}\right\}, \max _{i \in \Lambda}\left\{k_{2 i}\right\}\right\}$, then system (2) and system (3) achieve exponential synchronization under intermittent controller (4).

Proof. According to Lemma 1, we can choose a positive constant $\varepsilon>0$ such that

$$
r_{i}+p k_{1 i}-e^{\varepsilon \sigma} \sum_{j=1}^{n} a_{j i}-\varepsilon \geq 0, \mu_{i}+p k_{2 i}-e^{\varepsilon \sigma} \sum_{j=1}^{n} \xi_{j i}-\varepsilon \geq 0, i, j \in \Lambda .
$$

Let $e(t)=\left(e_{11}(t), e_{12}(t), \cdots, e_{1 n}(t), e_{21}(t), e_{22}(t), \cdots, e_{2 n}(t)\right)$, we can consider the Lyapunov-Krasovskii functional as follows:

$$
\begin{gathered}
V(t)=\sum_{i=1}^{n} e^{\varepsilon t}\left|e_{1 i}(t)\right|^{p}+\sum_{i=1}^{n} e^{\varepsilon t}\left|e_{2 i}(t)\right|^{p} \\
+e^{\varepsilon \sigma} \sum_{i=1}^{n} \sum_{j=1}^{n} a_{i j} \int_{t-\sigma(t)}^{t} e^{\varepsilon s}\left|e_{1 j}(t)\right|^{p} d s \\
+e^{\varepsilon \sigma} \sum_{i=1}^{n} \sum_{j=1}^{n} \xi_{i j} \int_{0}^{t} e^{\varepsilon s}\left|e_{2 j}(t)\right|^{p} d s .
\end{gathered}
$$

Form $T \leq t \leq(m+\theta) T$, we compute the upper right derivation of $V(t)$ along the solution of error system (5). From assumptions $\left(\mathrm{H}_{1}\right)-\left(\mathrm{H}_{4}\right)$, we obtain

$$
\begin{gathered}
\dot{V}(t) \leq \sum_{i=1}^{n}\left\{p e^{\varepsilon t}\left|e_{1 i}(t)\right|^{p-1}\left(-\left(1+k_{1 i}\right) e_{1 i}(t)+e_{2 i}(t)\right)+\varepsilon e^{\varepsilon t}\left|e_{1 i}(t)\right|^{p}\right. \\
+p e^{\varepsilon t}\left|e_{2 i}(t)\right|^{p-1}\left[\left|1-u_{i}\right|\left|e_{1 i}(t)\right|-\left(u_{i}-1+k_{2 i}\right)\left|e_{2 i}(t)\right|\right. \\
+h_{i}\left|e_{1 i}(t)\right| \\
+\bar{v}_{i}\left(\sum_{j=1}^{n}\left|c_{i j}\right| l_{j}\left|e_{1 j}(t)\right|\right)+\bar{v}_{i}\left(\sum_{j=1}^{n}\left|d_{i j}\right| l_{j}\left|e_{1 j}(t-\sigma(t))\right|\right) \\
\left.+s_{i}\left|e_{1 i}(t)\right|\left(\sum_{j=1}^{n}\left(\left|c_{i j}\right|+\left|d_{i j}\right|\right) m_{j}+\left|I_{i}\right|\right)\right] \\
+e^{\varepsilon \sigma} \sum_{j=1}^{n} a_{i j}\left(e^{\varepsilon t}\left|e_{1 j}(t)\right|^{p}-\left(1-\sigma_{1}\right) e^{\varepsilon(t-\sigma)}\left|e_{1 j}(t-\sigma(t))\right|^{p}\right)
\end{gathered}
$$




$$
\left.+e^{\varepsilon \sigma} \sum_{j=1}^{n} \xi_{i j} e^{\varepsilon t}\left|e_{2 j}(t)\right|^{p}\right\} .(7)
$$

According to Lemma 2, the following in equalities hold

$$
\begin{gathered}
p e^{\varepsilon t}\left|e_{2 i}(t)\right|\left|e_{1 i}(t)\right|^{p-1} \leq e^{\varepsilon t}\left(\left|e_{2 i}(t)\right|^{p}+(p-1)\left|e_{1 i}(t)\right|^{p}\right),(8) \\
p e^{\varepsilon t}\left|e_{2 i}(t)\right|^{p-1}\left[\left|1-u_{i}\right|+h_{i}\right]\left|e_{1 i}(t)\right| \\
\leq e^{\varepsilon t}\left[\left|1-u_{i}\right|+h_{i}\right]\left(\left|e_{1 i}(t)\right|^{p}+(p-1)\left|e_{2 i}(t)\right|^{p}\right), \\
p e^{\varepsilon t}\left|e_{2 i}(t)\right|^{p-1} s_{i}\left(\sum_{j=1}^{n}\left(\left|c_{i j}\right|+\left|d_{i j}\right|\right) m_{j}+\left|I_{i}\right|\right)\left|e_{1 i}(t)\right| \\
\leq e^{\varepsilon t} s_{i}\left(\sum_{j=1}^{n}\left(\left|c_{i j}\right|+\left|d_{i j}\right|\right) m_{j}+\left|I_{i}\right|\right)\left(\left|e_{1 i}(t)\right|^{p}+(p-1)\left|e_{2 i}(t)\right|^{p}\right), \\
p e^{\varepsilon t}\left|e_{2 i}(t)\right|^{p-1} \bar{v}_{i} \sum_{j=1}^{n}\left|c_{i j}\right| l_{j}\left|e_{1 j}(t)\right| \\
\leq \sum_{j=1}^{n} \bar{v}_{i}\left|c_{i j}\right| l_{j} e^{\varepsilon t}\left(\left|e_{1 j}(t)\right|^{p}+(p-1)\left|e_{2 i}(t)\right|^{p}\right)(11)
\end{gathered}
$$

$p e^{\varepsilon t}\left|e_{2 i}(t)\right|^{p-1} \bar{v}_{i} \sum_{j=1}^{n}\left|d_{i j}\right| l_{j}\left|e_{1 j}(t-\sigma(t))\right|$,

$$
\leq \sum_{j=1}^{n} \bar{v}_{i}\left|d_{i j}\right| l_{j} e^{\varepsilon t}\left(\left|e_{1 j}(t-\sigma(t))\right|^{p}+(p-1)\left|e_{2 i}(t)\right|^{p}\right) .
$$

By applying (8) - (12) and assumption $\left(\mathrm{H}_{5}\right)$ to $(7)$, one can deduce

$$
\begin{gathered}
\dot{V}(t) \leq \sum_{i=1}^{n} e^{\varepsilon t}\left[-p\left(1+k_{1 i}\right)+(p-1)+\left|1-u_{i}\right|+h_{i}\right. \\
\left.+s_{i}\left(\sum_{j=1}^{n}\left(\left|c_{i j}\right|+\left|d_{i j}\right|\right) m_{j}+\left|I_{i}\right|\right)+\sum_{j=1}^{n} \bar{v}_{j}\left|c_{j i}\right| l_{i}+e^{\varepsilon \sigma} \sum_{j=1}^{n} a_{j i}+\varepsilon\right]\left|e_{1 i}(t)\right|^{p} \\
+\sum_{i=1}^{n} e^{\varepsilon t}\left[-p\left(u_{i}-1+k_{2 i}\right)+1+(p-1)\left(\left|1-u_{i}\right|+h_{i}\right)\right. \\
\left.+(p-1) \sum_{j=1}^{n} \bar{v}_{i}\left(\left|c_{i j}\right|+\left|d_{i j}\right|\right) l_{j}+e^{\varepsilon \sigma} \sum_{j=1}^{n} \xi_{j i}+\varepsilon\right]\left|e_{2 i}(t)\right|^{p} \\
+\sum_{i=1}^{n} e^{\varepsilon t} \sum_{j=1}^{n}\left(\bar{v}_{i}\left|d_{i j}\right| l_{j}-\left(1-\sigma_{1}\right) a_{i j}\right)\left|e_{1 j}(t-\sigma(t))\right|^{p} \\
+\sum_{i=1}^{n} e^{\varepsilon t}\left(-r_{i}-p k_{1 i}+e^{\varepsilon \sigma} \sum_{j=1}^{n} a_{j i}+\varepsilon\right)\left|e_{1 i}(t)\right|^{p} \\
+\sum_{i=1}^{n} e^{\varepsilon t}\left(-\mu_{i}-p k_{2 i}+e^{\varepsilon \sigma} \sum_{j=1}^{n} \xi_{j i}+\varepsilon\right)\left|e_{2 i}(t)\right|^{p} \leq 0,(13)
\end{gathered}
$$

which implies that

$$
V(t) \leq V(m T), t \in[m T,(m+\theta) T) .
$$


If $(m+\theta) T \leq t \leq(m+1) T$, similarly, one has

$$
\begin{aligned}
& \dot{V}(t) \leq \sum_{i=1}^{n} e^{\varepsilon t}\left(-r_{i}-p k_{1 i}+e^{\varepsilon \sigma} \sum_{j=1}^{n} a_{j i}+\varepsilon\right)\left|e_{1 i}(t)\right|^{p}+\sum_{i=1}^{n} e^{\varepsilon t} p k_{1 i}\left|e_{1 i}(t)\right|^{p} \\
& +\sum_{i=1}^{n} e^{\varepsilon t}\left(-\mu_{i}-p k_{2 i}+e^{\varepsilon \sigma} \sum_{j=1}^{n} \xi_{j i}+\varepsilon\right)\left|e_{2 i}(t)\right|^{p}+\sum_{i=1}^{n} e^{\varepsilon t} p k_{2 i}\left|e_{2 i}(t)\right|^{p} \\
& \leq \sum_{i=1}^{n} p e^{\varepsilon t}\left(k_{1 i}\left|e_{1 i}(t)\right|^{p}+k_{2 i}\left|e_{2 i}(t)\right|^{p}\right) \leq p \varpi V(t),
\end{aligned}
$$

where $\varpi=\max \left\{\max _{i \in \Lambda}\left\{k_{1 i}\right\}, \max _{i \in \Lambda}\left\{k_{2 i}\right\}\right\}$, which leads to

$V(t) \leq V((m+\theta) T) \exp (p \varpi(t-m T-\theta T)), t \in[(m+\theta) T,(m+1) T)$.

Combining (14) and (16), we summarize that

$\left(\mathrm{P}_{1}\right)$ For $t \in[0, \theta T)$, it follows from (14) that

$$
V(t) \leq V(0) .
$$

$\left(\mathrm{P}_{2}\right)$ For $t \in[\theta T, T)$, from (16), we obtain that

$$
V(t) \leq V(\theta T) e^{p \varpi(t-\theta T)} \leq V(0) e^{p \varpi(t-\theta T)} .
$$

$\left(\mathrm{P}_{3}\right)$ For $t \in[T,(\theta+1) T)$, we have

$$
V(t) \leq V(T) \leq V(0) e^{p \varpi(1-\theta) T} .
$$

$\left(\mathrm{P}_{4}\right)$ For $t \in[(\theta+1) T, 2 T)$, we get that

$$
V(t) \leq V((1+\theta) T) e^{p \varpi(t-T-\theta T)} \leq V(0) e^{p \varpi(t-2 \theta T)} .
$$

Repeating this procedure, fort $\in[m T,(m+\theta) T)$, we obtain that

$$
V(t) \leq V(m T) \leq V(0) e^{m p \varpi(1-\theta) T} .
$$

Furthermore, for $t \in[(m+\theta) T,(m+1) T)$, we have

$$
V(t) \leq V((m+\theta) T) e^{p \varpi(t-m T-\theta T)} \leq V(0) e^{p \varpi(t-(m+1) \theta T)} .
$$

If $t \in[m T,(m+\theta) T)$, we can get $m \leq \frac{t}{T}$, then it follows from (17) that

$$
V(t) \leq V(0) e^{m p \varpi(1-\theta) T} \leq V(0) e^{p \varpi(1-\theta) t}
$$

Similarly, if $t \in[(m+\theta) T,(m+1) T)$, we can obtain $\frac{t}{T}<m+1$, from (18), it can be verified that

$$
V(t) \leq V(0) e^{p \varpi(t-(m+1) \theta T)} \leq V(0) e^{p \varpi(1-\theta) t} .
$$

Hence, for any $t \in[0,+\infty)$, we conclude

$$
V(t) \leq V(0) e^{p \varpi(1-\theta) t}
$$

From the definitions of $V(t)$ and (19), we derive that

$$
\begin{gathered}
\|e(t)\|_{p}^{p}=\sum_{i=1}^{n}\left(\left|e_{1 i}(t)\right|^{p}+\left|e_{2 i}(t)\right|^{p}\right) \leq e^{-\varepsilon t} V(t) \leq e^{-\varepsilon t} V(0) e^{p \varpi(1-\theta) t} \\
\leq \sum_{i=1}^{n}\left[\left|e_{1 i}(0)\right|^{p}+\left|e_{2 i}(0)\right|^{p}+e^{\varepsilon \sigma} \sum_{j=1}^{n} a_{i j} \int_{-\sigma(0)}^{0} e^{\varepsilon s}\left|e_{1 j}(s)\right|^{p} d s\right] e^{-(\varepsilon-p \varpi(1-\theta)) t}
\end{gathered}
$$




$$
\leq\left[1+\sigma e^{\varepsilon \sigma} \max _{1 \leq j \leq n}\left\{\sum_{i=1}^{n} a_{i j}\right)\right] \sum_{i=1}^{n} \sup _{-\sigma \leq s \leq 0}\left|\varphi_{i}(s)-\tilde{\varphi}_{i}(s)\right|^{p} e^{-(\varepsilon-p \omega(1-\theta)) t}
$$

$+\sum_{i=1}^{n} \sup _{-\sigma \leq s \leq 0}\left|\psi_{i}(s)-\tilde{\psi}_{i}(s)\right|^{p} e^{-(\varepsilon-p \varpi(1-\theta)) t}$

$\leq \Theta\|\Psi\|_{p}^{p} e^{-(\varepsilon-p \omega(1-\theta)) t}$,

Where $s \in[-\sigma, 0], \Theta=1+\sigma e^{\varepsilon \sigma} \max _{1 \leq j \leq n}\left\{\sum_{i=1}^{n} a_{i j}\right\} \geq 1$.

Therefore,

$$
\|e(t)\|_{p} \leq \Theta^{\frac{1}{p}}\|\Psi\|_{p} e^{-\left(\frac{\varepsilon}{p}-\omega(1-\theta)\right) t} .
$$

which means that the error system (5) is exponentially stable, i.e., the system (2) and system (3) achieved exponential synchronization under intermittent controller based on p-norm. The proof of Theorem 1 is completed.

Remark 1. Up to now, only a few literatures have applied periodic intermittent control to inertial neural networks, such as [22]-[23]. However, as far as we know, there is not exponential synchronization results of ICGNNs with time-varying delays by using periodic intermittent control, which shows that the results presented here are novel.

Remark2. In [24], based on the theory of infinite norms, the intermittent control has been employed to synchronize CGNNs systems. In this paper, the inertia term is not only introduced to the CGNNs model, which makes the model more complex, but also the synchronization of ICGNNs are analyzed according to the theory of p-norm. Compared with the reference [24], the above result is more general.

In system (2), for each $i \in \Lambda$, if the amplification function $v_{i}\left(x_{i}(t)\right)=v_{i}>0$, then system (1) can be simplified as the following form:

$$
\left\{\begin{aligned}
\frac{d x_{i}(t)}{d t}= & -x_{i}(t)+y_{i}(t) \\
\frac{d y_{i}(t)}{d t}= & -\left(1-u_{i}\right) x_{i}(t)-\left(u_{i}-1\right) y_{i}(t)-v_{i}\left[b_{i}\left(x_{i}(t)\right)\right. \\
& \left.-\sum_{j=1}^{n} c_{i j} f_{j}\left(x_{j}(t)\right)-\sum_{j=1}^{n} d_{i j} f_{j}\left(x_{j}(t-\sigma(t))\right)-I_{i}\right], t \geq 0
\end{aligned}\right.
$$

Evidently, in this case, the assumption $\left(\mathrm{H}_{1}\right),\left(\mathrm{H}_{2}\right)$ are satisfied. Correspondingly, assumptions $\left(\mathrm{H}_{3}\right)-\left(\mathrm{H}_{5}\right)$ can be stated as follows:

$\left(\widetilde{\mathrm{H}}_{3}\right)$ For any $x, z \in R$, there exists constants $l_{j}>0$ satisfying

$$
\left|f_{j}(x)-f_{j}(z)\right| \leq l_{j}|x-z|, j \in \Lambda \text {. }
$$

$\left(\widetilde{\mathrm{H}}_{4}\right)$ For any $x, z \in R, x \neq z$, there are constants $h_{i}>0$, such that

$$
\frac{v_{i}(z)-v_{i}(x)}{z-x} \geq h_{i}, i \in \Lambda \text {. }
$$

$\left(\widetilde{\mathrm{H}}_{5}\right)$ For every $i, j \in \Lambda, \tilde{r}_{i}+p k_{1 i}-\sum_{j=1}^{n} \tilde{a}_{j i}>0, \tilde{\mu}_{i}+p k_{2 i}-\sum_{j=1}^{n} \tilde{\xi}_{j i}>0$, where $\tilde{r}_{i}=1-\left|1-u_{i}\right|-v_{i} h_{i}-\sum_{j=1}^{n} v_{j}\left|c_{j i}\right| l_{i}$,

$$
\tilde{\mu}_{i}=p\left(u_{i}-1\right)-1-(p-1) \sum_{j=1}^{n} v_{i}\left(\left|c_{i j}\right|+\left|d_{i j}\right|\right) l_{j}
$$




$$
\begin{aligned}
& -(p-1)\left(\left|1-u_{i}\right|+v_{i} h_{i}\right), \\
\tilde{a}_{j i}= & \frac{v_{j}\left|d_{j i}\right| l_{i}}{1-\sigma_{1}}, \tilde{\xi}_{j i} \geq 0, \tilde{\xi}_{j i} \text { are some appropriately chosen numbers. }
\end{aligned}
$$

Similar to $\left(\mathrm{H}_{5}\right)$, there must be a suitable number $\tilde{\varepsilon}>0$ to satisfy

$$
\tilde{r}_{i}+p k_{1 i}-e^{\tilde{\varepsilon} \sigma} \sum_{j=1}^{n} \tilde{a}_{j i}-\tilde{\varepsilon} \geq 0, \tilde{\mu}_{i}+p k_{2 i}-e^{\tilde{\varepsilon} \sigma} \sum_{j=1}^{n} \tilde{\xi}_{j i}-\tilde{\varepsilon} \geq 0, i, j \in \Lambda .
$$

Meanwhile, the slave system (3) becomes

$$
\left\{\begin{aligned}
\frac{d z_{i}(t)}{d t}= & -z_{i}(t)+w_{i}(t)+K_{1 i} \\
\frac{d w_{i}(t)}{d t} & =-\left(1-u_{i}\right) z_{i}(t)-\left(u_{i}-1\right) w_{i}(t)-v_{i}\left[b_{i}\left(z_{i}(t)\right)\right. \\
& \left.-\sum_{j=1}^{n} c_{i j} f_{j}\left(z_{j}(t)\right)-\sum_{j=1}^{n} d_{i j} f_{j}\left(z_{j}(t-\sigma(t))\right)-I_{i}\right]+K_{2 i}
\end{aligned}\right.
$$

Where $K_{1 i}$ and $K_{2 i}$ remain the same as formula (4).

Therefore, according to Theorem 1, the following results are directly derived.

Corollary 1. Under assumptions $\left(\mathrm{H}_{2}\right),\left(\widetilde{\mathrm{H}}_{3}\right),\left(\widetilde{\mathrm{H}}_{4}\right),\left(\widetilde{\mathrm{H}}_{5}\right)$, system (21) and system (22) are exponential synchronization with the intermittent controller (4) if there exists $\tilde{\varepsilon}>0$ satisfying

$$
\frac{\tilde{\varepsilon}}{p}-\varpi(1-\theta)>0,
$$

and

$$
\tilde{r}_{i}+p k_{1 i}-e^{\tilde{\varepsilon} \sigma} \sum_{j=1}^{n} \tilde{a}_{j i}-\tilde{\varepsilon} \geq 0, \tilde{\mu}_{i}+p k_{2 i}-e^{\tilde{\varepsilon} \sigma} \sum_{j=1}^{n} \tilde{\xi}_{j i}-\tilde{\varepsilon} \geq 0, i, j \in \Lambda .
$$

Especially, for each $i \in \Lambda$, when the amplification function $v_{i}\left(x_{i}(t)\right)=1$, the system (2) is reduced to the following form:

$$
\left\{\begin{aligned}
\frac{d x_{i}(t)}{d t} & =-x_{i}(t)+y_{i}(t) \\
\frac{d y_{i}(t)}{d t} & =-\left(1-u_{i}\right) x_{i}(t)-\left(u_{i}-1\right) y_{i}(t)-b_{i}\left(x_{i}(t)\right) \\
& +\sum_{j=1}^{n} c_{i j} f_{j}\left(x_{j}(t)\right)+\sum_{j=1}^{n} d_{i j} f_{j}\left(x_{j}(t-\sigma(t))\right)+I_{i}
\end{aligned}\right.
$$

Accordingly, assumption $\left(\mathrm{H}_{5}\right)$ can be described as follows:

$\left(\widehat{H}_{5}\right)$ For every $i, j \in \Lambda, \hat{r}_{i}+p k_{1 i}-\sum_{j=1}^{n} \hat{a}_{j i}>0, \hat{\mu}_{i}+p k_{2 i}-\sum_{j=1}^{n} \hat{\xi}_{j i}>0$,

where

$$
\begin{gathered}
\hat{r}_{i}=1-\left|1-u_{i}\right|-h_{i}-\sum_{j=1}^{n}\left|c_{j i}\right| l_{i}, \\
\hat{\mu}_{i}=p\left(u_{i}-1\right)-1-(p-1) \sum_{j=1}^{n}\left(\left|c_{i j}\right|+\left|d_{i j}\right|\right) l_{j} \\
-(p-1)\left(\left|1-u_{i}\right|+h_{i}\right), \\
\hat{a}_{j i}=\frac{\left|d_{j i}\right| l_{i}}{1-\sigma_{1}}, \hat{\xi}_{j i} \geq 0, \hat{\xi}_{j i} \text { are some appropriately chosen numbers. }
\end{gathered}
$$

Then, the system (3) is degenerated to the following form: 


$$
\left\{\begin{aligned}
\frac{d z_{i}(t)}{d t}= & -z_{i}(t)+w_{i}(t)+K_{1 i} \\
\frac{d w_{i}(t)}{d t}= & -\left(1-u_{i}\right) z_{i}(t)-\left(u_{i}-1\right) w_{i}(t)-b_{i}\left(z_{i}(t)\right) \\
& +\sum_{j=1}^{n} c_{i j} f_{j}\left(z_{j}(t)\right)+\sum_{j=1}^{n} d_{i j} f_{j}\left(z_{j}(t-\sigma(t))\right)+I_{i}+K_{2 i}
\end{aligned}\right.
$$

Where $K_{1 i}$ and $K_{2 i}$ are the same as formula (4).

From Theorem 1, we can easily obtain the following corollary.

Corollary 2. Suppose that assumptions $\left(\mathrm{H}_{2}\right),\left(\widetilde{\mathrm{H}}_{3}\right),\left(\widetilde{\mathrm{H}}_{4}\right),\left(\widehat{\mathrm{H}}_{5}\right)$ hold. Then system $(23)$ and system (24) are exponential synchronization with the intermittent controller (4), if there exists $\hat{\varepsilon}>0$ meeting

$$
\frac{\hat{\varepsilon}}{p}-\varpi(1-\theta)>0
$$

and

$$
\hat{r}_{i}+p k_{1 i}-e^{\hat{\varepsilon} \sigma} \sum_{j=1}^{n} \hat{a}_{j i}-\hat{\varepsilon} \geq 0, \hat{\mu}_{i}+p k_{2 i}-e^{\hat{\varepsilon} \sigma} \sum_{j=1}^{n} \hat{\xi}_{j i}-\hat{\varepsilon} \geq 0, i, j \in \Lambda .
$$

Remark 3. When $\theta \rightarrow 1$, the periodic intermittent control (4) can be simplified to continuous control. In this case, it is obvious that the assumption $\left(\mathrm{H}_{5}\right)$ is satisfied. Hence, as long as the assumptions $\left(\mathrm{H}_{1}\right)-\left(\mathrm{H}_{4}\right)$ are true, we can ensure that the exponential synchronization of systems (2) and (3) can be achieved.

\section{Numerical simulations}

In this section, an example is presented to show the validity and feasibility of theoretical results presented in the previous section.

Example 1. Consider the following inertial Cohen-Grossberg with time-varying delays:

$$
\begin{array}{r}
\frac{d^{2} x_{i}(t)}{d t^{2}}=-u_{i} \frac{d x_{i}(t)}{d t}-v_{i}\left(x_{i}(t)\right)\left[b_{i}\left(x_{i}(t)\right)-\sum_{j=1}^{n} c_{i j} f_{j}\left(x_{j}(t)\right)\right. \\
\left.-\sum_{j=1}^{n} d_{i j} f_{j}\left(x_{j}(t-\sigma(t))\right)-I_{i}\right], i, j \in \Lambda,
\end{array}
$$

where $u_{1}=u_{2}=2, v_{i}(x)=0.3+\frac{0.1}{1+x^{2}}, b_{1}\left(x_{1}(t)\right)=0.7 x_{1}(t), b_{2}\left(x_{2}(t)\right)=0.8 x_{2}(t), c_{11}=d_{21}=$ $0.1, c_{12}=d_{22}=0.2, c_{21}=d_{11}=d_{12}=0.15, c_{22}=-0.05, f_{j}(x)=0.5 \tanh (x), I_{1}=I_{2}=1.5$ and $\sigma(t)=0.2 \sin (t)$.

By variable substitution, the system (25) can be transformed into a first-order form, and the corresponding master-slave systems are defined as same as system (2) and system (3), respectively. Evidently, for any $x, z \in R$, we obtain that

$$
0.3 \leq v_{i}(x) \leq 0.4,\left|v_{i}(x)-v_{i}(z)\right|=\left|\frac{0.1}{1+x^{2}}-\frac{0.1}{1+z^{2}}\right| \leq 0.05|x-z|, i=1,2,
$$

which shows that

$$
s_{1}=s_{2}=0.05 .
$$

And for any $x, z \in R, x \neq z$. we get that 


$$
\frac{v_{1}(z) b_{1}(z)-v_{1}(x) b_{1}(x)}{z-x} \geq 0.175, \frac{v_{2}(z) b_{2}(z)-v_{2}(x) b_{2}(x)}{z-x} \geq 0.2,
$$

which implies that

$$
h_{1}=0.175, h_{2}=0.2 \text {. }
$$

Moreover, we know that $\sigma=\sigma_{1}=0.2, m_{1}=m_{2}=0.5, l_{1}=l_{2}=0.5$ and $a_{11}=a_{12}=0.0375$, $a_{21}=0.025, a_{22}=0.05$. For $p=2$, selecting the appropriate constant $\xi_{11}=\xi_{12}=\xi_{21}=\xi_{22}=$ 0.1 and $k_{11}=k_{12}=2, k_{21}=k_{22}=1.5$, through simple calculation, it is easy to observe that hypothesis $\left(\mathrm{H}_{1}\right)-\left(\mathrm{H}_{5}\right)$ hold. It follows from assumption $\left(\mathrm{H}_{5}\right)$ that $\dot{\varepsilon}=3.4869, \grave{\varepsilon}=2.2771$, and $\varepsilon=\min \left\{\varepsilon^{\prime}, \dot{\varepsilon}\right\}=2.2771$. Correspondingly, we obtain $\theta>0.4307$ by computing. Taking the control period $T=1$ and $\theta=0.5$, obviously, all the conditions in Theorem 1 are satisfied. Therefore, the master-slave systems are exponentially synchronized under the designed control mechanism. State trajectories of variables $x_{1}, z_{1}, x_{2}, z_{2}$ and variables $y_{1}, w_{1}, y_{2}, w_{2}$ are shown in Fig.1 and Fig.2, respectively. The time evolutions of synchronization errors $e_{11}, e_{12}, e_{21}, e_{22}$ are given in Fig.3.
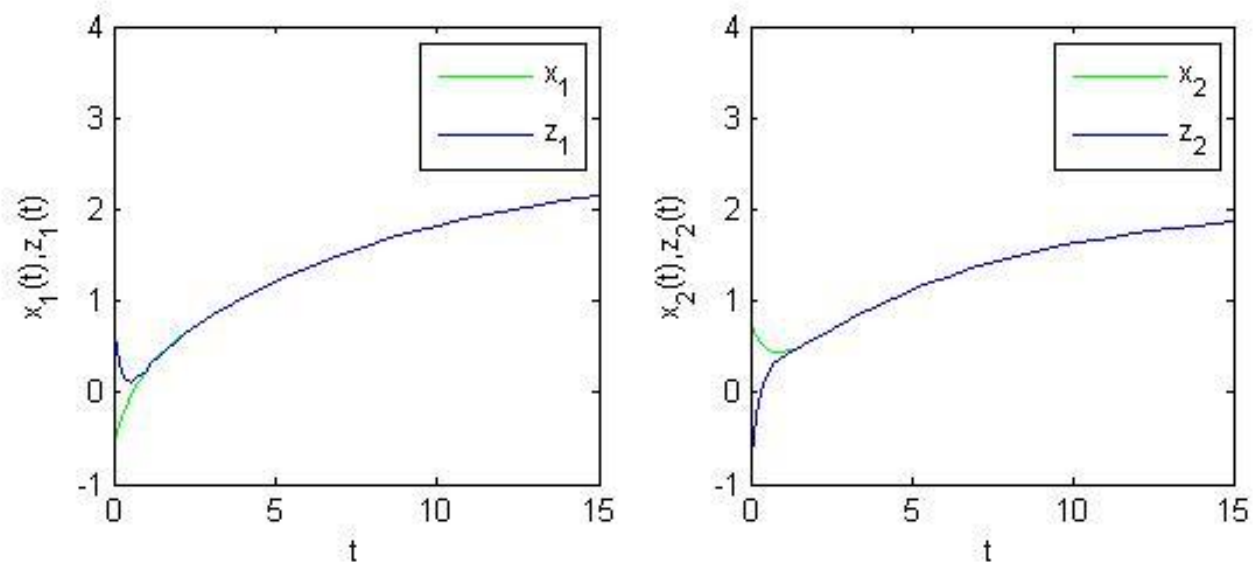

Fig.1 State trajectories of variables $x_{1}, z_{1}, x_{2}, z_{2}$ for the master-slave systems.
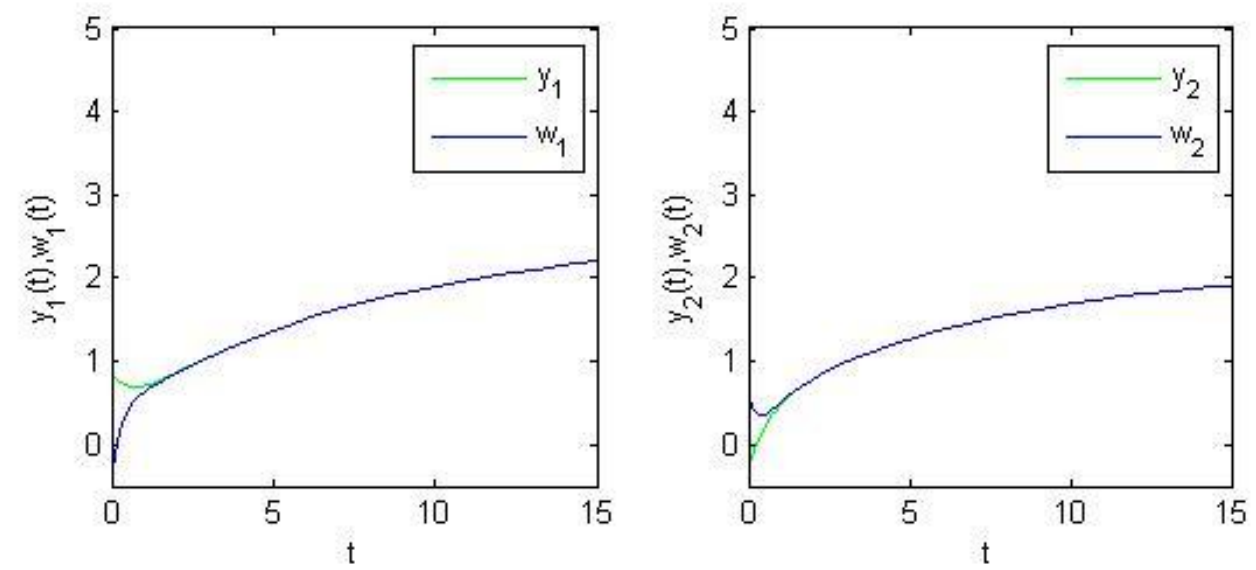

Fig.2 State trajectories of variables $y_{1}, w_{1}, y_{2}, w_{2}$ for the master-slave systems. 

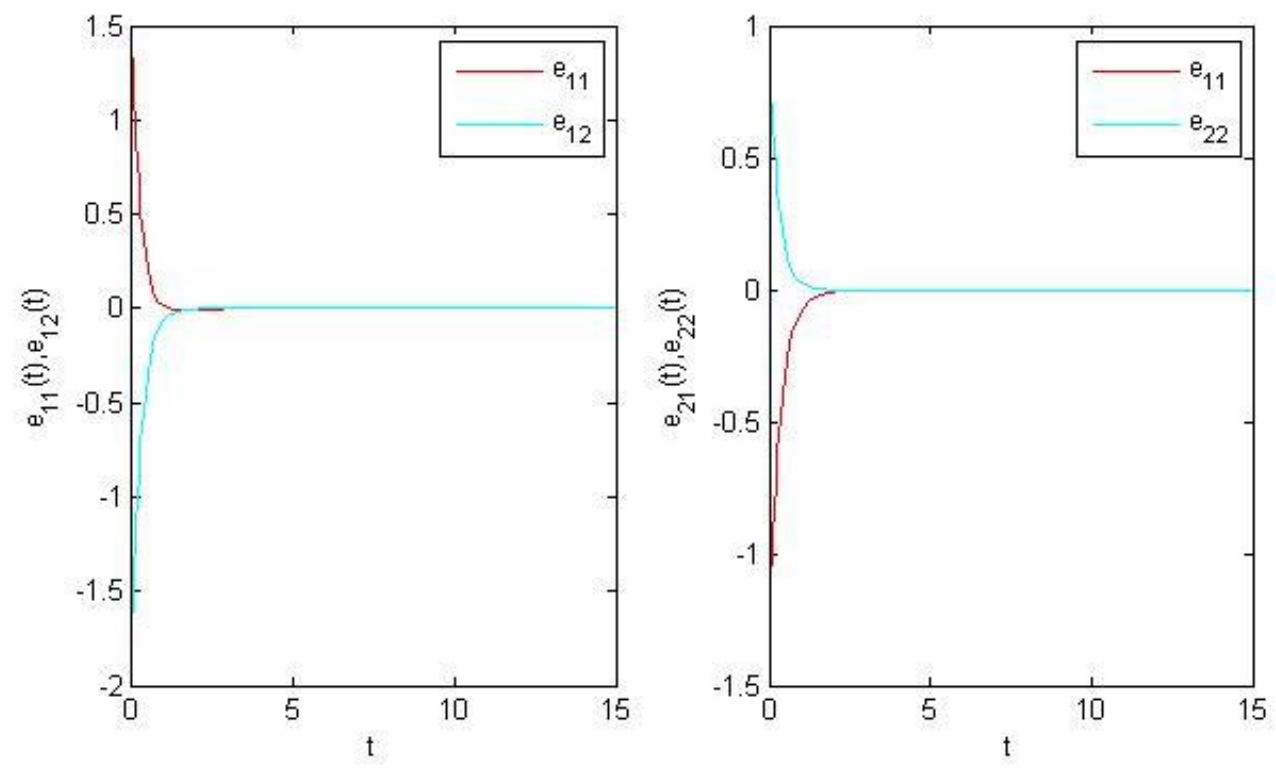

Fig.3 The time evaluations synchronization errors $e_{11}, e_{12}, e_{21}, e_{22}$.

\section{Conclusions}

In this paper, by using variable transformation, the inertial Cohen-Grossberg Neural Network model with time-varying delay is transformed into a first-order form. Moreover, by constructing the Lyapunov-Krasovskii functional and employing the inequality technique, some novel criteria on the exponential synchronization of the master systems and slave systems are presented under periodic intermittent control. The results we have obtained further improve the previous results since inertial terms are taken into account. In future, we will further investigate the synchronization problem of stochastic inertial Cohen-Grossberg Neural Networks with mixed delays.

\section{Acknowledgment}

The authors are grateful for the support of the National Natural Science Foundation of China (61503046, 61803046).

\section{References}

[1] M.A. Cohen, and S. Grossberg, "Absolute stability of global pattern formation and parallel memory storage by competitive neural networks," IEEE Trans. Syst. Man Cybern., vol. 13, no. 5, pp. 815-826, 1983.

[2] W. Jiang, L. Li, Z. Tu, and Y. Feng, "Lagrange Stability for Delayed-Impulses in Discrete-Time Cohen-Grossberg Neural Networks with Delays,” Neural Process Lett., vol. 51, no. 7, pp. 1835-1848, 2020.

[3] Z. Cai, and L. Huang, "Finite-time synchronization by switching state-feedback control for discontinuous Cohen-Grossberg neural networks with mixed delays," Int. J. Mach. Learn. Cybern.,vol. 9, no. 10, pp. 1683-1695, 2018.

[4] Y. Zhou, C. Li, L. Chen, and T. Huang, "Global exponential stability of memristive Cohen-Grossberg neural networks with mixed delays and impulse time window,” Neurocompuing, vol. 275, pp. 2384-2391, 2018.

[5] D. Peng, X. Li, C. Aouiti, and F. Miaadi, "Finite-time synchronization for Cohen-Grossberg neural networks with mixed time-delays". Neurocomputing, vol.294, pp. 39-47, 2018.

[6] R. Li, J. Cao, A. Alsaedi, and F. Alsaadi, "Exponential and fixed-time synchronization of Cohen-Grossberg neural networks with time-varying delays and reaction-diffusion terms," Appl. Math. Comput., vol. 313, pp. 37-51, 2017. 
[7] J.F. Ashmore, and D. Attwell, "Models for electrical tuning in hair cells," Proc. R. Soc. Lond. B, vol. 226, no. 1244, pp. 325-344, 1985.

[8] D.E. Angelaki, and M.J. Correia, "Models of membrane resonance in pigeon semicircular canal type II hair cells," Biol. Cybern., vol. 65,pp.1-10,1991.

[9] P.D. Mitcheson, T.C. Green, and E.M. Yeatman, "Power processing circuits for electromagnetic, electrostatic and piezoelectric inertial energy scavengers,” Microsyst. Technol., vol. 13,pp. 1629-1635, 2007.

[10] E.E. Aktakka, and K. Najafi, "A micro inertial energy harvesting platform with self-supplied power management circuit for autonomous wireless sensor nodes," IEEE J. Solid-State Circ., vol. 49, no. 9, pp. 2017-2029, 2014.

[11] Y. Ke, and C.Miao, "Exponential stability of periodic solutions for inertial Cohen-Grossberg-type neural networks," Neural Netw. World, vol.24, no. 4, pp. 377-394, 2014.

[12] C. Aouiti, E.A. Assali, and Y.E. Foutayeni, "Finite-Time and Fixed-Time Synchronization of Inertial Cohen - Grossberg-Type Neural Networks with Time Varying Delays,” Neural Process Lett., vol.50, no. 3, pp. 2407-2436, 2019.

[13] L. Ke, and W. Li, "Exponential synchronization in inertial Cohen - Grossberg neural networks with time delays," J. Frankl. Inst.-Eng. Appl. Math., vol.356, no. 18, pp. 11285-11304, 2019.

[14] C. Aouiti, and E.A. Assali, "Nonlinear Lipschitz measure and adaptive control for stability and synchronization in delayed inertial Cohen-Grossberg-type neural networks," Int. J. Adapt. Control Signal Process, vol.33, no. 10,pp.1457-1477,2019.

[15] S. Yu, Z. Zhang, and Z. Quan, "New global exponential stability conditions for inertial Cohen-Grossberg neural networks with time delays," Neurocomputing, vol.151, pp. 1446-1454, 2015.

[16] X. Li, J. Fang, and H. Li, "Master-slave exponential synchronization of delayed complex-valued memristor-based neural networks via impulsive control," Neural Netw., vol. 93, pp. 165-175, 2017.

[17] P. Yang, and X. Tang, "Exponential synchronization for neural networks with mixed time-varying delays via periodically intermittent control," Int. J. Biomath., vol. 7, no. 2, pp. 1-18, 2014.

[18] S. Li, X. Peng, Y. Tang, and Y. Shi, "Finite-time synchronization of time-delayed neural networks with unknown parameters via adaptive control," Neurocomputing, vol. 308, pp. 65-74, 2018.

[19] C. Guan, D. Sun, Z. Fei, and C. Ren, "Synchronization for switched neural networks via variable sampled-data control method," Neurocomputing, vol. 311, pp. 325-332, 2018.

[20] J. Xing, H. Jiang, and C. Hu, "Exponential synchronization for delayed recurrent neural networks via periodically intermittent control," Neurocomputing, vol. 113, pp. 122-129, 2013.

[21] G. Zhang, and Y. Shen, "Exponential synchronization of delayed memristor-based chaotic neural networks via periodically intermittent control," Neural Netw., vol. 55, pp. 1-10, 2014.

[22] Q. Tang, and J. Jian, "Exponential synchronization of inertial neural networks with mixed time-varying delays via periodically intermittent control,” Neurocomputing, vol. 338, pp.181-190,2019.

[23] P. Wan, D. Sun, D. Chen, M. Zhao, and L. Zheng, "Exponential synchronization of inertial reaction-diffusion coupled neural networks with proportional delay via periodically intermittent control,"Neurocomputing, vol.356, pp. 195-205,2019.

[24] J. Yu, C. Hu, H. Jiang, and Z. Teng, "Exponential synchronization of Cohen-Grossberg neural networks via periodically intermittent control,” Neurocomputing, vol.74, no. 10, pp. 1776-1782, 2011.

[25] J. Mei, M. Jiang, B. Wang, Q. Liu, W. Xu, and T. Liao, "Exponential p-Synchronization of Non-autonomous Cohen-Grossberg Neural Networks with Reaction-Diffusion Terms via Periodically Intermittent Control," Neural Process Lett., vol.40, no. 2, pp. 103-126, 2014.

[26] M. Hui, N. Luo, H. Iu, Q. Wu, R. Yao, and L. Bai, "Pinning Synchronization via Intermittent Control for 
Memristive Cohen-Grossberg Neural Networks With Mixed Delays,” IEEE Access, vol.8, pp. 55676-55687, 2020. 